tivbegehren (§56 JN)“ und vom „alternativ (§ $56 \mathrm{JN})$ ) erhobenen Ersatzbegehren, jedoch an keiner Stelle davon, dass die beklagte Partei zwischen Herausgabe und Zahlung wählen könne. Aber ungeachtet dessen haben die Kläger ein echtes Alternativbegehren nicht erhoben. Ihr dazu erstattetes Vorbringen macht trotz der missglückten Formulierung unmissverständlich deutlich, dass sie primär die Herausgabe der Zeichnung begehren und im Fall der Erfolglosigkeit dieses Begehrens, weil das Werk in Verlust geraten ist, Schadenersatz wollen. So schreiben sie, dass die beklagte Partei ihnen "gegebenenfalls" den Geldersatz für den Verlust des Kunstwerks schulde und der Mittelwert als zu ersetzender Schaden heranzuziehen wäre, könnte sie das Werk nicht herausgeben. Auch wenn das Erstgericht das Herausgabebegehren als "Mehrbegehren" abwies, ging es doch in der Sache offensichtlich davon aus, dass die Kläger ein Haupt- und ein Eventualbegehren erhoben haben.
Entgegen der Auffassung des Berufungsgerichts ist das Eventualbegehren nicht unschlüssig. Die vermissten Angaben zur Bemessung des Werts der Zeichnung sind zur ausreichenden Behauptung der Schadenshöhe nicht notwendig. Hierzu genügt im Anlassfall die Benennung des Künstlers, der Werkgattung, des Sujets des Werks und des erzielbaren Verkaufserlöses. Diese Behauptungen stellten die Kläger auf.

Das Berufungsgericht wird daher im fortgesetzten Verfahren die aufgrund einer nicht zutreffenden Rechtsansicht nicht erledigten Rügen der Berufung der beklagten Partei zu behandeln und eine neuerliche Entscheidung zu treffen haben.

Die Kostenentscheidung beruht auf $\S 52$ Abs. 1 ZPO. (Entscheidung von der Redaktion bearbeitet.)

\title{
Zu den Voraussetzungen eines Rückgabeanspruchs nach § 6 Abs. 2 Kulturgü- terrückgabegesetz (KultGüRückG)
}

Bayerischer Verwaltungsgerichtshof, Beschluss vom 13. April 2010 - 7 CE 10.258

1. Bloße behördeninterne Ersuchen - etwa über Interpol gestellte Rechtshilfeersuchen mit einer Kulturgüter-Auflistung - genügen nicht der Bestimmung des § 6 Abs. 2 Satz 3 des Kulturgüterrückgabegesetzes (KultGüRückG), derzufolge das Verzeichnis der bedeutenden Kulturgüter im Bundesgebiet ohne unzumutbare Hindernisse öffentlich zugänglich sein muss.

2. Solchen behördeninternen Ersuchen ist nicht nur der Wortlaut des Kulturgüterrückgabegesetzes, sondern auch das Interesse des Kunsthandels an Rechtssicherheit entgegenzuhalten.

3. §12 Abs. 1 Nr. 2 KultGüRückG vermittelt den zuständigen Behörden des ersuchenden Staates keinen Anspruch auf Begutachtung der streitgegenständlichen Kulturgüter. (Leitsätze der Redaktion)

\section{Tenor}

Die Beschwerde wird zurückgewiesen.

\section{Gründe}

\section{I.}

- Die Antragstellerin begehrt im Wege einer einstweiligen Anordnung die Anhaltung derzeit in Bayern befindlicher archäologischer Fundstücke des guatemaltekischen Kulturerbes nach dem Kulturgüterrückgabegesetz.

Aufgrund einer Beschlagnahmeanordnung der Staatsanwaltschaft München I, bestätigt durch Beschluss des Amtsgerichts München vom 24. April 2008, wurde am 23. April 2008 in den Räumlichkeiten einer Münchner Spedition eine große Anzahl archäologischer Fundstücke (so genannte "Sammlung Patterson") beschlagnahmt. Der Beschlagnahme lag ein internationales Rechtshilfeersuchen wegen des Verdachts der illegalen Einfuhr der Kunstsammlung nach Deutschland durch den
Beigeladenen zugrunde. Die Fundstücke kamen aus Santiago de Compostela, Spanien, wo sie bereits 1997 Teil der Ausstellung "der Geist des prähispanischen Amerikas, 3000 Jahre Kultur" waren. Am 22. Juni 2009 wurde der Botschaft der Antragstellerin in Deutschland über das Auswärtige Amt eine CD des Bayerischen Landeskriminalamts mit einer Bestandsaufnahme und einem Fotoregister der sichergestellten Stücke übergeben. Daraufhin bat die Antragstellerin auf diplomatischem Wege beim Auswärtigen Amt der Bundesrepublik Deutschland wiederholt um die Rückgabe von 369 Objekten, die Teil des prähispanischen Kulturerbes von Guatemala seien.

Am 13. Oktober 2009 beantragte die Antragstellerin beim Bayerischen Verwaltungsgericht München, den Antragsgegner im Wege einer einstweiligen Anordnung zu verpflichten, die Anhaltung der 369 im einzelnen aufgelisteten archäologischen Fundstücke, die gemeinsam mit anderen archäologischen Fundstücken am 23. April 2008 beschlagnahmt wurden, anzuordnen. Es bestehe der dringende Verdacht, dass die Kulturgüter unrechtmäßig aus der Republik Guatemala ausgeführt worden seien. Zudem bestehe der für eine Anhaltung erforderliche 
Rückgabeanspruch nach dem Kulturgüterrückgabegesetz. Die Anordnung der Anhaltung sei dringlich, da die Beschlagnahme der Sammlung voraussichtlich am 14. Oktober 2009 aufgehoben werde.

Mit Beschluss vom 14. Oktober 2009 verpflichtete das Verwaltungsgericht München den Antragsgegner, bis zu einer Entscheidung des Gerichts im anhängigen Verfahren die beantragte Anhaltung anzuordnen. Diesem Beschluss kam das Bayerische Staatsministerium für Wissenschaft, Forschung und Kunst am 16. Oktober 2009 nach. Mit Beschluss vom gleichen Tage hob das Amtsgericht München die Beschlagnahme vom April 2008 auf, da die Voraussetzungen der Beschlagnahme für Zwecke der internationalen Rechtshilfe in strafrechtlichen Angelegenheiten entfallen seien.

Mit Beschluss vom 25. Januar 2010 lehnte das Verwaltungsgericht München den Antrag auf Erlass einer einstweiligen Anordnung ab. Die Voraussetzungen für die Anordnung der Anhaltung nach $\S 8$ Abs. 2 des Kulturgüterrückgabegesetzes (KultGüRückG) lägen nach summarischer Prüfung nicht vor. Zwar bestehe der dringende Verdacht der unrechtmäßigen Verbringung der streitgegenständlichen Kulturgüter in das Bundesgebiet. Die Antragstellerin habe jedoch keinen Rückgabeanspruch gemäß $§ 6$ Abs. 2 KultGüRückG glaubhaft machen können. Die Antragstellerin habe die archäologischen Fundstücke nicht binnen Jahresfrist vor ihrer Verbringung nach Spanien bzw. in die Bundesrepublik Deutschland als besonders bedeutsam im Sinne des $\S 6$ Abs. 2 Satz 2 KultGüRückG bezeichnet. Es handle sich auch nicht um vor ihrer Verbringung unbekannte Gegenstände, die binnen Jahresfrist nach Kenntnisnahmemöglichkeit von ihrer Existenz als besonders bedeutsam bezeichnet worden seien. Insbesondere reiche hierfür der Beschluss des guatemaltekischen Ministeriums für Kultur und Sport vom 24. September 2009 nicht aus. Am 24. September 2009 sei die Jahresfrist bereits abgelaufen gewesen, für deren Beginn die Möglichkeit der Erlangung der Kenntnis von den Kulturgütern maßgeblich sei. Bereits 1997 seien die archäologischen Fundstücke im Rahmen einer öffentlich zugänglichen Ausstellung gezeigt worden; noch dazu in Kombination mit dem Vorhandensein eines Ausstellungskataloges hätten die Behörden des Herkunftsstaates die Möglichkeit einer Kenntnisnahme gehabt. Zudem hätte im Jahr 2007 erneut die Möglichkeit bestanden, von den Gegenständen Kenntnis zu nehmen. Die spanische Polizei habe nämlich bereits im Februar 2007 die gesamte in Spanien lagernde "Sammlung Patterson" in einer Lichtbildmappe zusammengestellt sowie eine CD erstellt und via Interpol allen betroffenen Ländern zur Verfügung gestellt. Auf dieser CD seien bis auf die Fundstücke Foto Nr. 456, LKA Nr. 205 und Foto Nr. 1431, LKA Nr. 378 alle weiteren von der Antragstellerin geforderten Gegenstände enthalten. Weiterhin dürften ausweislich der Verwaltungsakten die Informationen der spanischen Behörden letztlich auch Basis eines offensichtlich bereits seit 2007 anhängigen Verfahrens 1.409-2007 vor dem Gericht erster Instanz in Strafsachen, für Rauschgifttaten und Straftaten gegen die Umwelt Sacatepequez sein. Zudem habe die Antragstellerin selbst im April 2008 via Interpol ein Schreiben an die deutschen Behörden übermittelt, welches ebenfalls bereits eine Auflistung eines Großteils der streitgegenständlichen Fundstücke (ca. 300) enthalte; bezüglich dieser sei mithin nicht nur von einer Möglichkeit der Kenntnisnahme, sondern von Kenntnis seitens der guatemaltekischen Behörden auszugehen.

Soweit überhaupt noch angenommen werden könne, dass bezüglich der streitgegenständlichen Kulturgüter im September 2009 die Möglichkeit bestanden hätte, sie noch „nachträglich" als besonders bedeutsam zu bezeichnen, sei diese nicht genutzt worden. Die laut Antragstellerin durch Beschluss des Kultur- und Sportministeriums vom 24. September 2009 durch Aufnahme in das Verzeichnis der Kulturgüter des Staates Guatemala vorgenommene nachträgliche Bezeichnung als besonders bedeutsam entspreche nicht den Anforderungen des $\S 6$ Abs. 2 Satz 2, 3 KultGüRückG. Insbesondere sei weder dargelegt noch nachgewiesen worden, dass das betreffende Verzeichnis im Bundesgebiet frei zugänglich sei.

Gegen diesen Beschluss wendet sich die Antragstellerin mit ihrer Beschwerde. Nach § 6 Abs. 2 Satz 1 Nr. 1 und 2 KultGüRückG sei jeder Gegenstand geschützt, der vor seiner Verbringung als besonders bedeutsam bezeichnet worden sei. Hierbei genüge es, wenn das Verfahren zur Bezeichnung eingeleitet sei, sofern dies öffentlich bekannt gemacht worden sei. Art. 7 des UNESCO-Übereinkommens über Maßnahmen zur Verhinderung der verbotenen Einfuhr, Ausfuhr und Eigentumsübertragung von Kulturgütern fordere lediglich die Eintragung des zurückzuführenden Objekts in der Inventarliste der betroffenen Institution. Zwar werde die Errichtung eines nationalen Verzeichnisses empfohlen; dies bleibe allerdings jedem Staat freigestellt, sei also keine bindende Verpflichtung. Die Antragstellerin führe ein nationales Verzeichnis, in dem u.a. auch alle archäologischen Ausgrabungen aufgeführt seien. Die Antragstellerin habe auch nicht im Februar 2007 in Form einer von der spanischen Polizei gefertigten Lichtbildmappe Kenntnis erhalten. Die Antragstellerin sei im Jahr 2007 durch den Kurator des Museo del Hombre Dominicano benachrichtigt worden, dass die Polizei in Galizien, in einem vom Beigeladenen gemieteten Depot, eine Vielzahl von prähispanischen Fundstücken sichergestellt habe. Der Kurator habe dieser Benachrichtigung Fotografien von 491 prähispanischen Fundstücken beigefügt. Anhand dieser Fotos hätten erstmals 124 der 491 Fundstücke als Teil des guatemaltekischen Maja-Kulturerbes identifiziert werden können. Diese Stücke seien am 24. September 2007 vom Leiter des Registers der Kulturschätze der Generaldirektion des Kultur- und Naturerbes beim Kultur- und Sportministerium vorläufig registriert worden. Zudem sei festgestellt worden, dass noch andere Fundstücke existierten, deren Echtheit jedoch noch an Ort und Stelle hätte überprüft werden müssen. Zu einer Begutachtung sei es jedoch nicht mehr gekommen, da die Fundstücke illegal aus Spanien verbracht worden seien. Bis heute sei nicht geklärt worden, inwieweit es sich bei den streitgegenständlichen 369 Fundstücken tatsächlich um Originale handle. Es entspreche dem Sinn des Gesetzes und dem Geist 
der UNESCO-Konvention, bis zur endgültigen Klärung der Authentizität der Objekte von dem Erfordernis abzusehen, diese Objekte in ein öffentlich zugängliches Verzeichnis einzutragen bzw. die Frist für eine solche Eintragung angemessen zu verlängern.

Von offizieller Stelle habe die Antragstellerin erstmals am 19. Juni 2009 durch die Übergabe der CD des Landeskriminalamts eine Dokumentation der sichergestellten Fundstücke erhalten. Bereits im Jahr 2007 seien Rechtshilfeanträge und zwar auch über Interpol gestellt worden, in denen zumindest 123 Fundstücke als Kulturgut im Sinne der UNESCO-Konvention erklärt und damit auch öffentlich als solche bekannt gegeben worden seien. Dabei handle es sich um die Fundstücke, die an Hand der 491 Fotografien als voraussichtliche Originale identifiziert hätten werden können.

Was das Erfordernis der öffentlichen Zugänglichkeit eines Verzeichnisses angehe, sei dies nach einhelliger Expertenmeinung derzeit völlig unrealistisch. Aus Sicherheits- und konservatorischen Gründen hielten die Museen und Institutionen ihre vollständigen Verzeichnisse größtenteils unter Verschluss. Die Sicherheit, die durch die Aufnahme in ein öffentliches Verzeichnis gewährleistet werden solle, sei vorliegend bereits durch die Rechtshilfeanträge der Staatsanwaltschaft Guatemala an die spanischen und deutschen Behörden, denen entsprechende Verzeichnisse beigefügt gewesen seien, gegeben gewesen. Im Ergebnis werde das Kulturgüterrückgabegesetz in seiner derzeitigen Form Fällen wie dem vorliegenden in keiner Weise gerecht.

\section{Die Antragstellerin beantragt,}

den Beschluss des Bayerischen Verwaltungsgerichts München vom 25. Januar 2010 aufzuheben und den Antragsgegner zu verpflichten, die Anhaltung der im Verzeichnis des archäologischen Gutachtens vom 7. August 2009 angeführten 369 archäologischen Fundstücke des guatemaltekischen Kulturerbes, die gemeinsam mit anderen archäologischen Fundstücken im April 2008 von den deutschen Zollbehörden beschlagnahmt wurden, anzuordnen.

\section{Der Antragsgegner beantragt,}

die Beschwerde zurückzuweisen.

Nicht das Kulturgüterübereinkommen, sondern das Kulturgüterrückgabegesetz sei die hier maßgebliche deutsche Rechtsgrundlage. Deren Auslegung durch das Verwaltungsgericht sei nicht unvertretbar restriktiv. Es bestehe kein Anspruch der Antragstellerin auf Begutachtung. § 12 Abs. 1 KultGüRückG sei eine reine Aufgabennorm. Aus Art. 5 Buchstabe b des UNESCO-Übereinkommens vom 14. November 1970 ergebe sich ausdrücklich und unmissverständlich die Pflicht, ein Verzeichnis des national wertvollen Kulturguts in jedem Vertragsstaat zu führen. Der Antragsgegner habe weder von dem ausdrücklich in $\S 6$ Abs. 2 Satz 3 KultGüRückG normierten Erfordernis eines öffentlich zugänglichen Verzeichnisses absehen können, noch die ebenfalls ausdrücklich geregelte Frist für eine nachträgliche Eintragung nach eigenem Ermessen verlängern können. Jedenfalls sei die Jahresfrist ab möglicher Kenntnisnahme nicht eingehalten. Zudem seien an die öffentliche Bekanntmachung der Einleitung des Bezeichnungsverfahrens im Interesse der Rechtssicherheit als Ratio der Norm dieselben Anforderungen zu stellen wie an die Publizität eines Verzeichnisses gemäß § 6 Abs. 2 Satz 2 KultGüRückG. Die Bekanntmachung müsste daher im Bundesgebiet ohne unzumutbare Hindernisse öffentlich zugänglich sein, insbesondere käme eine Einstellung in das Internet in Betracht.

Entgegen der Auffassung der Antragstellerin gehe es dem Kunsthandel um Rechtssicherheit dahingehend, ab einem gewissen Zeitpunkt nicht mehr dem Risiko von Rückgabeansprüchen ausländischer Staaten ausgesetzt zu sein. Absurd sei die Auffassung, wonach mit der Übermittlung eines Rechtshilfeersuchens samt Auflistung der zurückgeforderten Gegenstände über Interpol an deutsche Behörden dem Erfordernis eines öffentlichen Verzeichnisses Genüge getan sei.

Der Beigeladene schließt sich den Ausführungen des Antragsgegners an und beantragt, die Beschwerde zurückzuweisen.

Wegen der weiteren Einzelheiten des Sach- und Streitstandes wird auf die Inhalt der Gerichtsakten und der vorgelegten Verwaltungsakten Bezug genommen.

II.

Die zulässige Beschwerde ist nicht begründet.

1.

Das Verwaltungsgericht hat den Antrag nach § 123 Abs. 1 VwGO zu Recht abgelehnt, da die Antragstellerin einen Anordnungsanspruch auf Anhaltung archäologischer Fundstücke nach dem Kulturgüterrückgabegesetz nicht glaubhaft machen konnte (§ 123 Abs. 3 VwGO iVm § 920 Abs. 2, § 294 ZPO). Die dargelegten und vom Senat allein geprüften Beschwerdegründe (vgl. § 146 Abs. 4 Satz 6 VwGO) rechtfertigen keine andere Entscheidung.

Nach § 8 Abs. 2 Satz 1 des Kulturgüterrückgabegesetzes (KultGüRückG) ordnen die zuständigen Behörden die Anhaltung von Kulturgut an, wenn der dringende Verdacht besteht, dass es unrechtmäßig aus einem anderen Mitgliedsstaat der Europäischen Union oder Vertragsstaat in das Bundesgebiet verbracht worden und an diesen Staat zurückzugeben ist. Gemäß § 8 Abs. 5 Satz 4 KultGüRückG ist der Rückgabeanspruch glaubhaft zu machen. Nach summarischer Prüfung fehlt es im vorliegenden Fall jedoch an der Glaubhaftmachung eines Rückgabeanspruchs nach $\S 6$ Abs. 2 KultGüRückG. Diese Bestimmung lautet: 
„(2) Ein unrechtmäßig nach dem 26. April 2007 aus dem Hoheitsgebiet eines Vertragsstaats in das Bundesgebiet verbrachter Gegenstand ist dem Vertragsstaat auf sein Ersuchen zurückzugeben, wenn

1. dieser Gegenstand vor der Verbringung oder im Fall von archäologischen Gegenständen, die vor der Verbringung unbekannt waren, innerhalb eines Jahres, nachdem die zuständige Behörde des betroffenen Vertragsstaats von dem Gegenstand Kenntnis erlangen konnte, von dem ersuchenden Vertragsstaat aus religiösen oder weltlichen Gründen als für die Archäologie, Vorgeschichte, Geschichte, Literatur, Kunst oder Wissenschaft besonders bedeutsam bezeichnet wurde oder ein Verfahren zur Bezeichnung eingeleitet und die Einleitung des Verfahrens öffentlich bekannt gemacht wurde und

2. der Gegenstand einer der in Art. 1 des Kulturgutübereinkommens genannten Kategorien angehört.

Als "besonders bedeutsam bezeichnet" im Sinne von Satz 1 Nr. 1 gilt ein Gegenstand, wenn er individuell identifizierbar von einem anderen Vertragsstaat in ein Verzeichnis des bedeutenden öffentlichen und privaten Kulturgutes aufgenommen worden ist. Das Verzeichnis muss im Bundesgebiet ohne unzumutbare Hindernisse öffentlich zugänglich sein. Lässt sich nicht klären, ob ein Gegenstand, der vor dem 26. April 2007 als besonders bedeutsam im Sinne des Satzes 1 Nr. 1 bezeichnet worden ist, vor oder nach diesem Tag ins Bundesgebiet verbracht worden ist, so gilt er als nach diesem Tag ins Bundesgebiet verbracht."

Das Verwaltungsgericht ging zu Recht davon aus, dass die Voraussetzungen für einen Rückgabeanspruch nach dieser Vorschrift in mehrerlei Hinsicht nicht erfüllt sind.

a. Die im vorliegenden Verfahren maßgebliche Rechtsgrundlage ist das Kulturgüterrückgabegesetz. Die völkerrechtlichen Verpflichtungen aus dem UNESCO-Kulturgutübereinkommen vom 14. November 1970 (vgl. Gesetz zu dem Übereinkommen vom 14.11.1970 über Maßnahmen zum Verbot und zur Verhütung der rechtswidrigen Einfuhr, Ausfuhr und Übereignung von Kulturgut vom 20. April 2007, BGBI. II S. 626) sind nicht unmittelbar anwendbar, sondern wurden durch das Kulturgüterrückgabegesetz vom 18. Mai 2007 (BGBI. I 2007, 757) in nationales Recht umgesetzt (vgl. dazu Begründung zum Gesetzentwurf BT-Drs. 16/1371 S. 12). Im Übrigen stützt auch die Antragstellerin selbst ihren geltend gemachten Anspruch ausdrücklich auf § 8 Abs. 2 iVm § 6 Abs. 2 KultGüRückG. Demgegenüber wäre dem UNESCO-Übereinkommen auch keine konkrete, unmittelbar anwendbare Anspruchsgrundlage zu entnehmen.

Zwar ist nach allgemeiner Meinung eine völkerrechtsfreundliche Interpretation des nationalen Rechts geboten (vgl. Jarass in: Jarass/Pieroth, GG, 10. Aufl. 2009, Rn. 4 zu Art. 25 mwN), d.h. dass Gesetze möglichst im Einklang mit den völkerrechtlichen
Verträgen auszulegen sind (vgl. auch Herdegen, Völkerrecht, 7. Aufl. 2008, Rn. 21 zu § 22 mwN). Jedoch darf im Wege der Auslegung einem nach Wortlaut und Sinn eindeutigen Gesetz nicht ein entgegengesetzter Sinn verliehen, der normative Gehalt der auszulegenden Norm nicht grundlegend neu bestimmt und das gesetzgeberische Ziel nicht in einem wesentlichen Punkt verfehlt werden (BVerfG vom 11. Juni 1980 - 1 PBvU 1/79= BVerfGE 54, 277 [299]).

b. Die streitgegenständlichen Kulturgüter sind nicht binnen eines Jahres nach Möglichkeit der Kenntnisnahme im Sinne des § 6 Abs. 2 Satz 1 Nr. 1, Satz 2 KultGüRückG als besonders bedeutsam bezeichnet worden.

Wie das Verwaltungsgericht in der angefochtenen Entscheidung zutreffend ausgeführt hat, läuft die Jahresfrist von der Möglichkeit der Kenntnisnahme an. Dies ergibt sich auch bei völkerrechtsfreundlicher Interpretation des Gesetzes (vgl. oben a]) aus dem eindeutigen und unmissverständlichen Wortlaut des $\S 6$ Satz 1 Abs. 2 Nr. 1 KultGüRückG („innerhalb eines Jahres, nachdem die zuständige Behörde [...] Kenntnis erlangen konnte"). Bereits im Jahr 1997 waren nahezu alle streitgegenständlichen Kulturgüter in Spanien im Rahmen der Ausstellung „Der Geist des prähispanischen Amerikas, 3000 Jahre Kultur" im Museo do Pobo Galego und in der Kirche San Domingos de Banaval gezeigt bzw. im dazugehörigen Ausstellungskatalog abgebildet worden. Damit war für die Antragstellerin die Möglichkeit einer Kenntnisnahme gegeben. Eine weitere umfassende Möglichkeit der Kenntnisnahme bot sich für die Antragstellerin im Jahre 2007. Im Februar 2007 hatte die spanische Polizei nämlich die gesamte "Sammlung Patterson" in einer Lichtbildmappe zusammengestellt und auf dem Interpolwege an alle betroffenen Länder übermittelt (vgl. BI. 300 der Verwaltungsakten). Zwar bestreitet die Antragstellerin dies, da in dem Vermerk der spanischen Polizei die „beteiligten Staaten“ nicht explizit benannt worden seien. Jedoch gibt es keinen Anhaltspunkt dafür, dass und warum gerade die Antragstellerin nicht zu den Adressaten dieser Übermittlung gehört haben soll. Zudem räumt die Antragstellerin selbst ein, dass sie im Jahr 2007 durch den Kurator des „Museo del Hombre Dominicano“ unter Übersendung der Fotografien von 491 prähispanischen Fundstücken von der Sicherstellung einer Vielzahl prähispanischer Fundstücke durch die spanische Polizei unterrichtet wurde. Ferner führt die Antragstellerin in ihrer Beschwerdebegründung selbst aus, dass ein Teil (124) dieser 491 Fundstücke am 24. September 2007 unter der Amtsnummer 39-07/PH vom Leiter des Registers der Kulturschätze der Generaldirektion des Kultur- und Naturerbes beim Kultur- und Sportministerium vorläufig registriert worden sei; ein Antrag auf Überprüfung anderer Fundstücke sei bereits 2007 durch die Staatsanwaltschaft der Republik Guatemala bei den spanischen Behörden gestellt worden. Schließlich übermittelte die Antragstellerin den deutschen Behörden via Interpol ein Schreiben der Staatsanwaltschaft Guatemala vom 24. April 2008 (Bl. 45 ff. der Verwaltungsakten), das sich mit einem aus Spanien ausgeführten Posten von ca. 300 Gegenständen befasst, die Teil des Kulturerbes der Republik Guatemala seien. 
Zudem wurde in diesem Schreiben mitgeteilt, dass hinsichtlich dieser ca. 300 Gegenstände offenbar bereits 2007 ein gerichtliches Verfahren wegen illegaler Ausfuhr von Kulturgütern und anderem (vgl. „Causa 1.409-2007") eingeleitet wurde (BI. 46 der Verwaltungsakten). Demgemäß ist nicht nur von einer Möglichkeit der Kenntnisnahme, sondern sogar von Kenntnis der Antragstellerin auszugehen.

c. Die Kulturgüter wurden bis heute auch noch nicht "als besonders bedeutsam bezeichnet" im Sinne des § 6 Abs. 2 Satz 2 KultGüRückG.

Entgegen der Auffassung der Antragstellerin genügen die im Jahr 2007 auch über Interpol gestellten Rechtshilfeersuchen mit einer Auflistung der Kulturgüter nicht diesen Anforderungen. Nach § 6 Abs. 2 Satz 3 KultGüRückG muss das Verzeichnis der bedeutenden Kulturgüter im Bundesgebiet ohne unzumutbare Hindernisse öffentlich zugänglich sein. Davon kann bei behördeninternen Ersuchen nicht die Rede sein.

Die Aufnahme der 369 Fundstücke in das Register der Kulturgüter der Republik Guatemala durch den Beschluss des Kulturund Sportministeriums vom 24. September 2009 erfolgte zum einen bereits nach Ablauf der Jahresfrist und entspricht auch im Übrigen nicht den Anforderungen des § 6 Abs. 2 Satz 2, 3 KultGüRückG. Wie das Verwaltungsgericht bereits im Einzelnen ausgeführt hat, hat die Antragstellerin weder dargelegt noch nachgewiesen, dass das betreffende Verzeichnis im Bundesgebiet ohne unzumutbare Hindernisse öffentlich zugänglich ist. Darauf kann Bezug genommen werden. Da es jedenfalls an der öffentlichen Zugänglichkeit des Verzeichnisses im Bundesgebiet fehlt, kann dahingestellt bleiben, ob die Abfassung des Verzeichnisses in Spanisch der Anforderung des $§ 6$ Abs. 2 Satz 3 KultGüRückG „ohne unzumutbare Hindernisse“ entspricht (vgl. dazu BT-Drs. 16/1371 S. 18, rechte Spalte).

Was die in der Beschwerdebegründung dargestellte, allerdings nicht näher substantiierte und in der ersten Instanz noch gar nicht erwähnte vorläufige Registrierung vom 24. September 2007 (vgl. oben 1. b]) angeht, hat auch die Antragstellerin selbst nicht dargelegt, dass diese im Bundesgebiet öffentlich zugänglich sei (vgl. § 6 Abs. 2 Satz 3 KultGüRückG) oder dass wenigstens die Einleitung des Verfahrens zur Bezeichnung als besonders bedeutsam öffentlich bekannt gemacht worden sei (vgl. dazu Rietschel Internationale Vorgaben zum Kulturgüterschutz und ihre Umsetzung in Deutschland, 2009, S. 141).

Soweit die Antragstellerin einwendet, die Sicherheit, die durch die Aufnahme in ein öffentliches Verzeichnis gewährleistet werden solle, sei bereits durch die Rechtshilfeanträge der Staatsanwaltschaft Guatemala mit entsprechenden Verzeichnissen gegeben gewesen, ist dem zum Einem der Wortlaut des Kulturgüterrückgabegesetzes entgegenzuhalten und zum Anderen das vom Antragsgegner dargestellte Interesse des Kunsthandels an Rechtssicherheit. Die Abwägung zwischen den Interessen des Kulturgutschutzes und den berechtigten Interessen des Kunsthandels hat der Gesetzgeber vorgenommen (vgl. BT-Drs. 16/1371 S. 12, linke Spalte).

Die Antragstellerin kann auch mit ihrem Einwand, der deutsche Gesetzgeber gehe mit seinen Anforderungen an ein Verzeichnis der Kulturgüter über die Regeln der UNESCOKonvention hinaus, nicht durchdringen. Zum Einen sind die Bestimmungen des primär maßgeblichen Kulturgüterrückgabegesetzes (vgl. oben 1. a]) eindeutig und unmissverständlich. Zum Anderen ergibt sich aus Art. 5 Buchstabe b des UNESCOÜbereinkommens vom 14. November 1970 (vgl. BGBI. 2007 II S. 630) die Verpflichtung der Vertragsstaaten, ein nationales Bestandsverzeichnis des bedeutsamen öffentlichen und privaten Kulturguts zu führen.

d. Schließlich vermittelt § 12 Abs. 1 Nr. 2 KultGüRückG der Antragstellerin auch keinen Anspruch auf Begutachtung der streitgegenständlichen Kulturgüter. Abgesehen davon, dass diese Bestimmung der Antragstellerin schon nach ihrem Wortlaut keinen Anspruch vermittelt, könnten damit auch nicht die gesetzlichen Voraussetzungen der § 8 Abs. 2 bzw. § 6 Abs. 2 KultGüRückG modifiziert werden.

\section{2.}

Nach alldem konnte die Beschwerde nicht zum Erfolg führen und war mit der Kostenfolge des § 154 Abs. 2 VwGO zurückzuweisen. Die Antragstellerin hat billigerweise auch die außergerichtlichen Kosten des Beigeladenen zu erstatten, zumal dieser durch seine Antragstellung selbst ein Kostenrisiko einging ( $\mathrm{vgl}$. $\S 162$ Abs. 3, § 154 Abs. 3 VwGO).

Die Streitwertfestsetzung ergibt sich aus $\S 47$, $§ 53 \mathrm{Abs} .3 \mathrm{Nr}$. $1 \mathrm{iVm} \S 52$ Abs. 2 GKG.

Dieser Beschluss ist unanfechtbar, § 152 Abs. 1 VwGO. (Entscheidung von der Redaktion bearbeitet.) 